\title{
Knowledge Upgrade to Wisdom by Using Knowledge Broker Agent for Web Application, Based on Users' Experiences
}

\author{
Hasanen S. Abdullah and Roaad A. Ali \\ Department of Computer Sciences, University of Technology, Baghdad-Iraq. \\ Corresponding Author: roaad-abed@yahoo.com.
}

\begin{abstract}
Knowledge is an important factor in conduct individual's life and decision-making process, so decision's efficiency and power is dependent on knowledge deepen which is possess by individual. The aim of this paper is to build an improved intelligent web application system to provide web service which upgrades and raises knowledge level to wisdom level by aggregate knowledge with experience. The web service is implemented by using the brokerage approach through agent-based knowledge broker web service system, and it related to pre-define field. Java Agent DEvelopment (JADE) platform is used in this system to provide the demand environment for knowledge broker agent to achieve its behaviors. Practical testing by submitting different query belong to the medical field (radiography) demonstrate the system efficiency in retrieving knowledge relevant to submitted query and provide level of knowledge upgraded to wisdom to sustain and support specialist users' capabilities in making decisions reach wise decisions level. [DOI: $10.22401 /$ JNUS.20.4.20]
\end{abstract}

Keywords: Knowledge, Information Broker, JADE platform, Knowledge Broker (KBr), Data Warehouse (DW), Enterprise Web Application.

\section{Introduction}

For any enterprise information resources are greatly valuable, these resources because of their importance they must be properly stored and easily accessible when they are required [1]. Human beings are more and more reliant on technology systems in the modern, data-driven era. Such technology needs to support transparent, aware decision-making processes in order to gain users' trust. Intelligent systems study and develop algorithms that implement the different learning models and their application to practical problems resolve [2]. These systems allow documentation of one or more expert knowledge and utilize the knowledge for problem solving in cost effective manner [3]. Information and Knowledge are important issue in decision making process. Simplification and evaluation of knowledge requires judgment, which few researchers have investigated. The judgment, selection and use of specific knowledge for a specific context are what is termed wisdom. That is, wisdom is related to the ability to efficiently choose and apply the appropriate knowledge in a certain state [4]. The Internet progress is leading to a number of new developments in software technology. One of the major issues is agent technology [4]. Information Broker (IB) is one of the main discovery and synchronization mechanisms among autonomous agents. Because of its properties and its wide applicability, a broker would be a natural candidate component for the web services infrastructure, and is widely used in distributed information systems such as Multi-agent systems and distributed databases [5]. One of the more significant new developments in Information system (IS) field is Data warehousing (DW). DW is wide in range has many mechanisms for preparing data to make it accessible for different end user tools for analysis and support decision making process [6].

\section{Background Theory}

In this section a background of most important concepts which is used to achieve this work.

\subsection{Knowledge Concept}

Knowledge can be defined as an expertise, and skills acquired by a person through education or experience [7]. It is benefit for refining our lives and makes or rises value for the enterprise in the context of business [1]. Using the knowledge relates to its application in organizational policy and practice. This 
involves taking and shaping decisions, making informed actions and modifying behaviors in order to achieve goals [8].

\subsection{Wisdom}

Wisdom is defined by Webster dictionary (1961) as the faculty of making the best use of knowledge, experience, and understanding by exercising good judgment. Learning is the input side of wisdom, and it can be defined as acquisition of knowledge, experience, and skills [1].

\subsection{Data Warehouse (DW)}

Data warehouse is considered as the basis of all decision support system (DSS) processing. Usually DSS analyst who works with the data warehouse environment is much easier than the analyst who works with the classical legacy environment because he deals with a single integrated source of data, granular data can be accessed easily, in addition, the data warehouse forms a basis for reusability and reconciliation of data. DW is defined as a subject oriented, integrated, nonvolatile, and time variant collection of data to support management's decisions [9].

\subsection{Information Broker (IB)}

IB is normally an individual on demand seeks to answer questions using all available sources and skilled in a number of areas that combined; provide the best chance of obtaining the information which are needed. IB provides services to researchers, policymakers, system analysts, architects, planners, and else who are acutely aware of the need of information and complexity of obtaining it [10]. Information broker has an important role in the mediation process between the users of information and information resources. IB is a professional in information services; IBs are both users of information environment and contributors to the information environment, because the information entity which is created by an IB is inserted to the information environment as secondary information and may serve other clients as well. IB possess properties enable it in succession in his profession such as:

1. Survey, scan and search the information sources, by exploring all of the components of Information environment.
2. Collect and store the obtained information and to structure, analyze, summarize and visualize it in a form suitable to serve the client [11].

\subsection{Software Agent}

Software agent is basically a software program designed to perform specific goaldriven functions and has the capability to communicate with other agents. The agent is able to ask for information and/or a decision from other and is also able to respond to events as they occur, modifying its behavior as required [12]. Multi-Agent Systems (MASs) are computational systems in which a collection of loosely-coupled autonomous agents interact in order to solve a given problem [13].

\subsection{Java Agent DEvelopment Framework (JADE)}

JADE is used by a heterogeneous community of users as a tool for both supporting research activities and building real applications, because JADE is a software framework facilitate the development of interoperable intelligent multi-agent systems. The goal of JADE is to simplify development while ensuring standard compliance through a comprehensive set of system services and agents, JADE provide number of facilities to agent programmer in order to perform that goal [14].

\subsection{Enterprise Web Application}

A web application is a client-server application. Client is a web browser that is run on user machine, so the users access business logic via their browsers. More companies are finding a way to offer their services and products online. When the web application distributed over a network is considered as an enterprise web application [15]. Publishing information on the web, make it accessible to anyone with access to the web, or using the web as a source of information to derive new knowledge [16].

\section{Related Works}

The most important researches that have related to the proposed system in this paper are illustrated: Joep Crompvoets et al [17], present a work in which Geospatial Information 
Broker (GIB) acts as an intermediary between service provider and users to facility a transaction between them to provide information that can be relied on by users in their decisions making. James Conklin et al [18], present knowledge broker in Seniors Health Research Transfer Network (SHRTN) has a crucial role in facilitate the transfer and exchange of knowledge (tacit and explicit knowledge) a cross social boundaries, so knowledge broker is act as bridge builder between those social boundaries; researchers, policy makers, caregivers, and decision makers to resolve work-related challenges in decision-making process. Anu Purhonen et al [19], present a semantic Information Broker (SIB) to manage runtime performance in smart environment, where SIB is using to share information among agents at heterogeneous device. David Budgen et al [20], develop a healthcare information broker prototype to support decision making process, where information broker retrieves and integrates information from autonomous and heterogeneous sources.

\section{Proposed Knowledge Upgrading Approach to Wisdom}

This work is dedicated to present the proposed wise knowledge broker; in this type of brokers, the knowledge gained from different supervised learning methodologies is combined with the experience gained by practicing deploying the knowledge broker in certain fields (i.e., this work has determined the field of retrieving medical images).

The proposed work is designed and implemented through three stages; first stage represents building the infrastructure of the $\mathrm{KBr}$. Stage two represents knowledge find out in web pages up to certain field by web page mining. In stage three $\mathrm{KBr}$ will use stage two to retrieve and display knowledge (images), in addition to that, in this stage $\mathrm{KBr}$ will acquire the experience and build the experience database (DW) to be a wise knowledge broker. Fig.(1) illustrates the skeletal scheme of the proposed work in which knowledge is revealed and how it merges with experience. Those stages are listed as follows: 


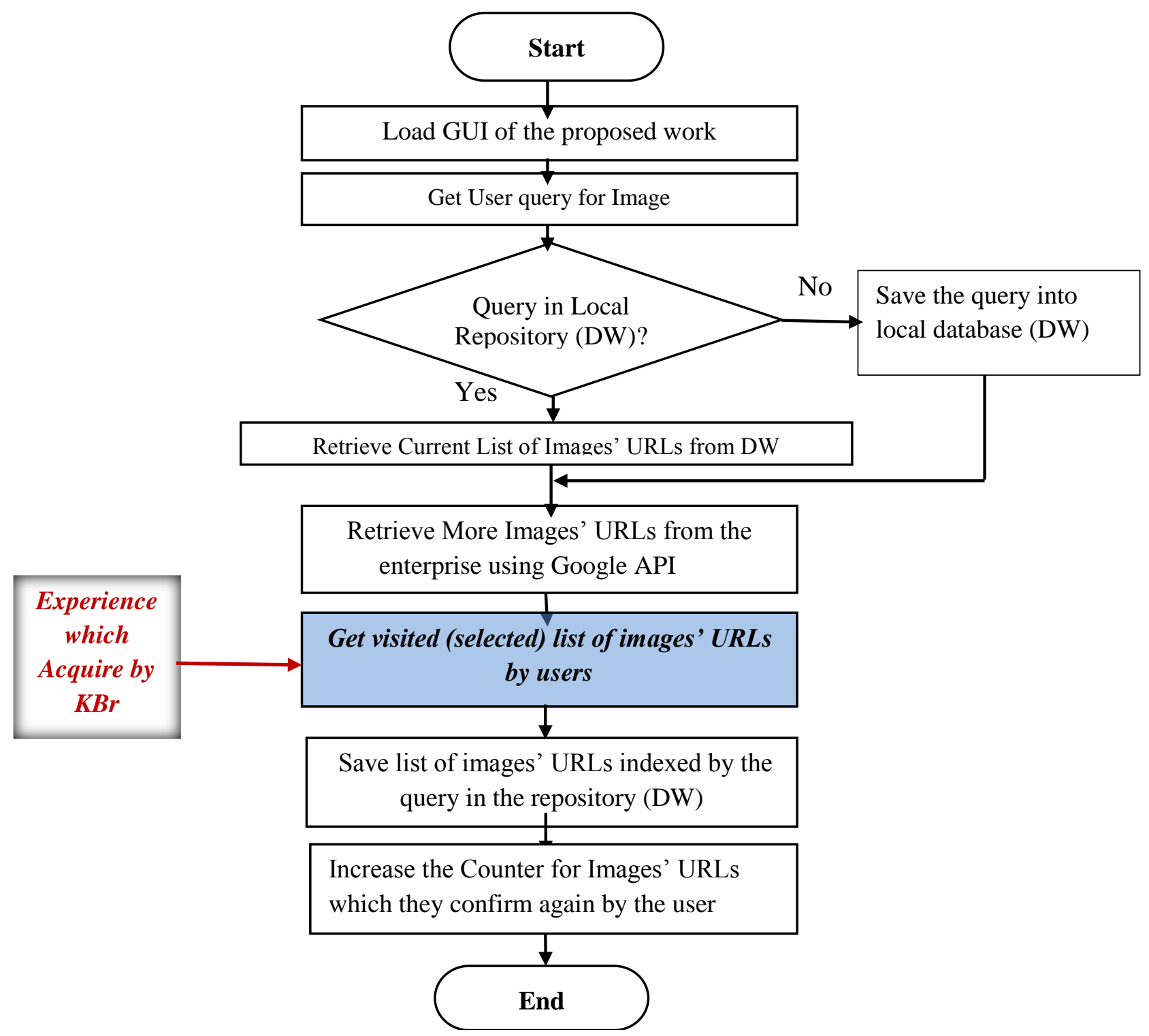

Fig.(1) : Flow Chart of Collecting Experience from Users by Binding Images' URLs with Queries.

\section{1 Building the Infrastructure}

This stage represents an implementation for building the infrastructure of the $\mathrm{KBr}$. $\mathrm{KBr}$ consist of two agents; first agent (active agent) is java script agent-based broker which is embedded within the web page (Graphical User Interface (GUI) of the proposed work). This agent is designed and implemented by HTML5 web worker files and it is activated whenever the proposed work GUI is loaded. It is responsible for dealing with the user.

Second agent (passive agent) is java agentbased broker which lived in JADE platform. The process of binding java agent which lived in JADE platform with the proposed web application that host in web server must be implemented, in order to enable the web application for communication with java agent-based broker. Java agent-based broker is responsible for implementing query process, discover and retrieve knowledge. In addition to present knowledge integrated with experience from DW. Flowchart in Fig.(2) illustrates the implementation of binding procedure of java agent-based broker with web application.

\subsection{Knowledge Find out}

In this stage, $\mathrm{KBr}$ is ready to receive and process the submitted query by the user. $\mathrm{KBr}$ begins the search process in the enterprise environment according to submitted query, knowledge is revealed in the medical field (radiography) according to the proposed work by implementing web page content mining process and this is achieved through mining in the content of this page which retrieved according to the query. 
Web pages are mined by the java agentbased broker to retrieve images (knowledge) by looking for specific tags (i.e., img, imgUrl) by following certain rules; these tags refer to links of images that can be retrieved corresponding to certain query. Fig.(3) presents the flowchart for extracting images' $\boldsymbol{U} \boldsymbol{R} \boldsymbol{L} \boldsymbol{s}$ within a web page.

$\mathrm{KBr}$ in this stage has the ability to read the site and retrieve the web page by Google API in addition to enable the java agent-based broker for reading content of the retrieved web page. Tokenized process which is implemented in retrieved page is achieved by using XML parser to identify appropriate parts of document to tokenize.
After tokenizing process, java agent-based broker will implement the inference token for valid image URLs which are relevance to query based on the correlation between the query and image description context, where with each image tag there is a text connect with it which considered as an annotation (description) for this image. When the web page content mining process is completed, list of images' URLs retrieved and sent to the front page at client side to java script agentbased broker which is responsible for displaying this list of images' URLs.

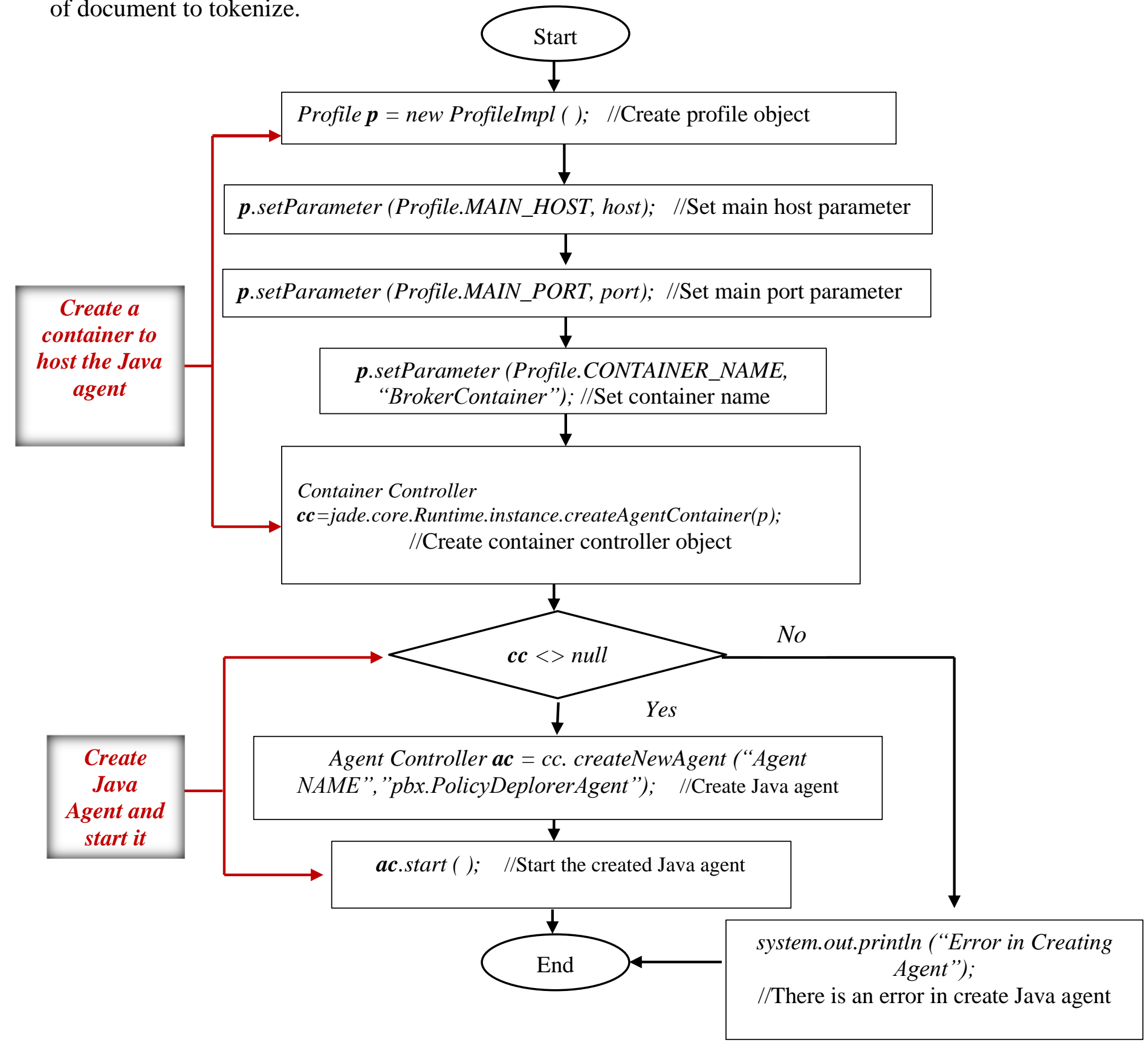

Fig.(2) : Flow Chart of Implement Procedure of Binding Java Agent with Web Application. 


\subsection{Retrieve Knowledge and Acquire the Experience}

In this stage of the proposed work design's stages, Java agent-based $\mathrm{KBr}$ uses the second stage of the design to possess the access ability to the web page according to submitted query by the user and retrieve knowledge (images' URLs tags) by following knowledge retrieval rules, which are adopted in this proposed work and display it to the user. Multiple users are submitting their queries against which search is conducted over the internet and list of images are retrieved and pushed to client side; at this point users starts visiting (select) images to sustain its fulfillment to the query they submit in the first place (as shown in the shaded rectangle in Fig.(1)); this behavior represent the experience of each user in determining group of images that can be tagged by the query; this experience is delivered to the backend modules of the proposed work and merged with others' experiences in the DW to promote decision capability.

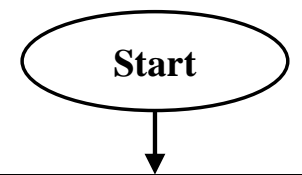

Post Query to the enterprise using Google API

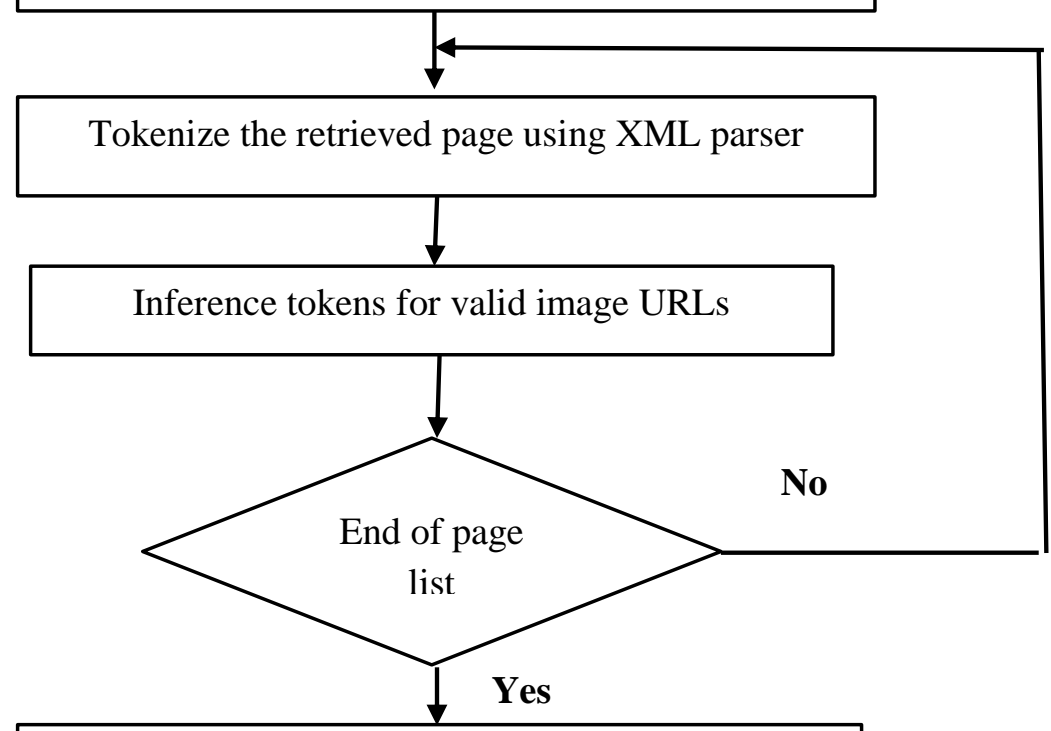

Posted retrieved images list to front page

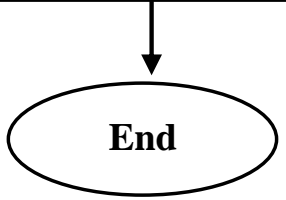

Fig.(3) : Flow Chart of Mining Retrieved Web Pages for Images' URLs.

As a result, the level which of the proposed system operates is making the data warehouse more efficient for the users by providing knowledge integrated with experience to support and elaborate users' capabilities in decision-making process to make the wise decisions.

\section{Knowledge Upgrading Approach to Wisdom Results}

The proposed work is tested by submitting different queries which belong to the medical field. The results of testing the proposed system are explained and illustrated when the query "feet" is submitted to the KBr. Fig.(4) shows the GUI of the proposed work, and shows "feet" as submitted query by the user. 


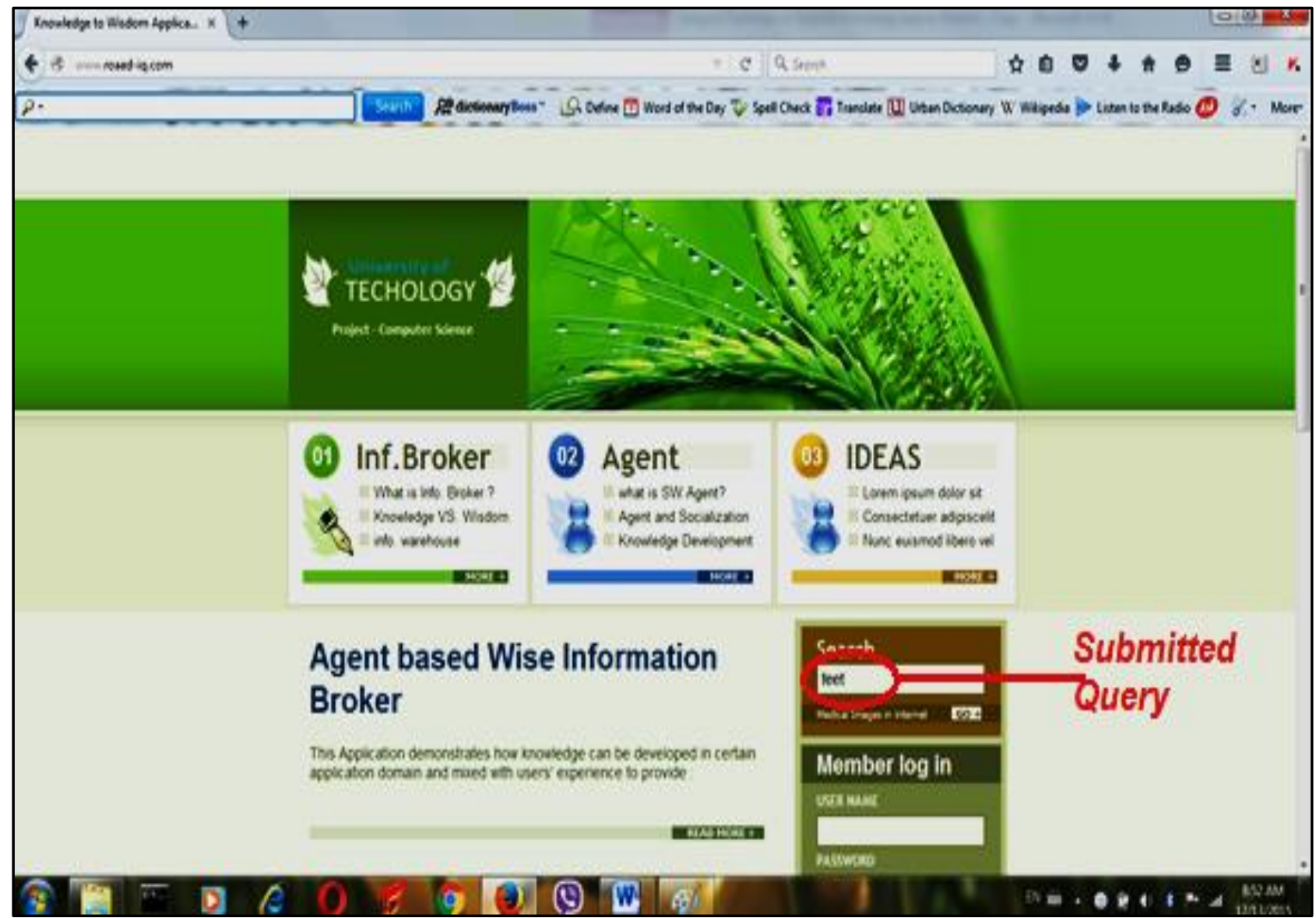

Fig.(4) : Submitted Query as an Example of "feet".

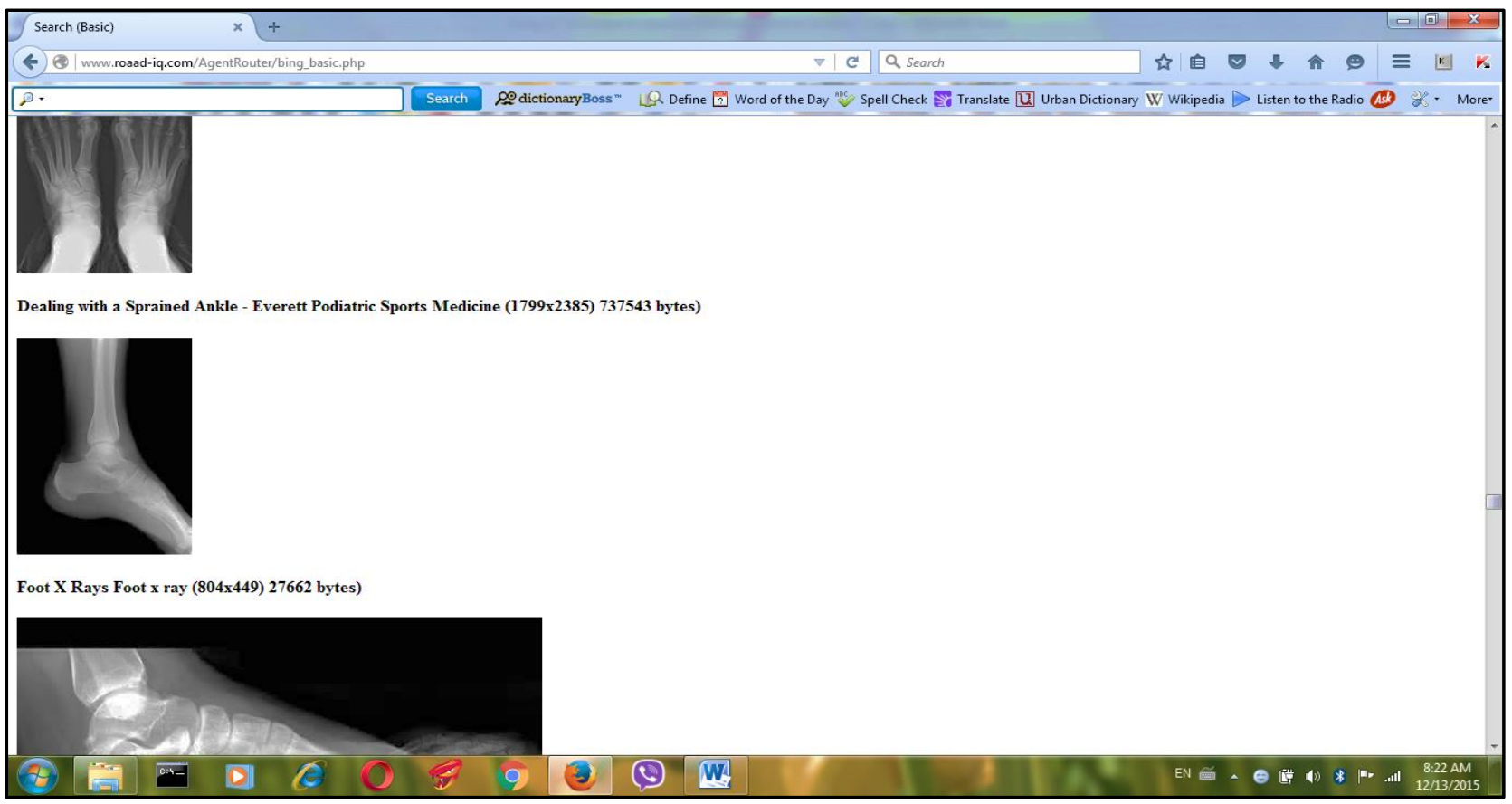

Fig.(4 a) : Query “feet” Results for Proposed work (Web Searching. 


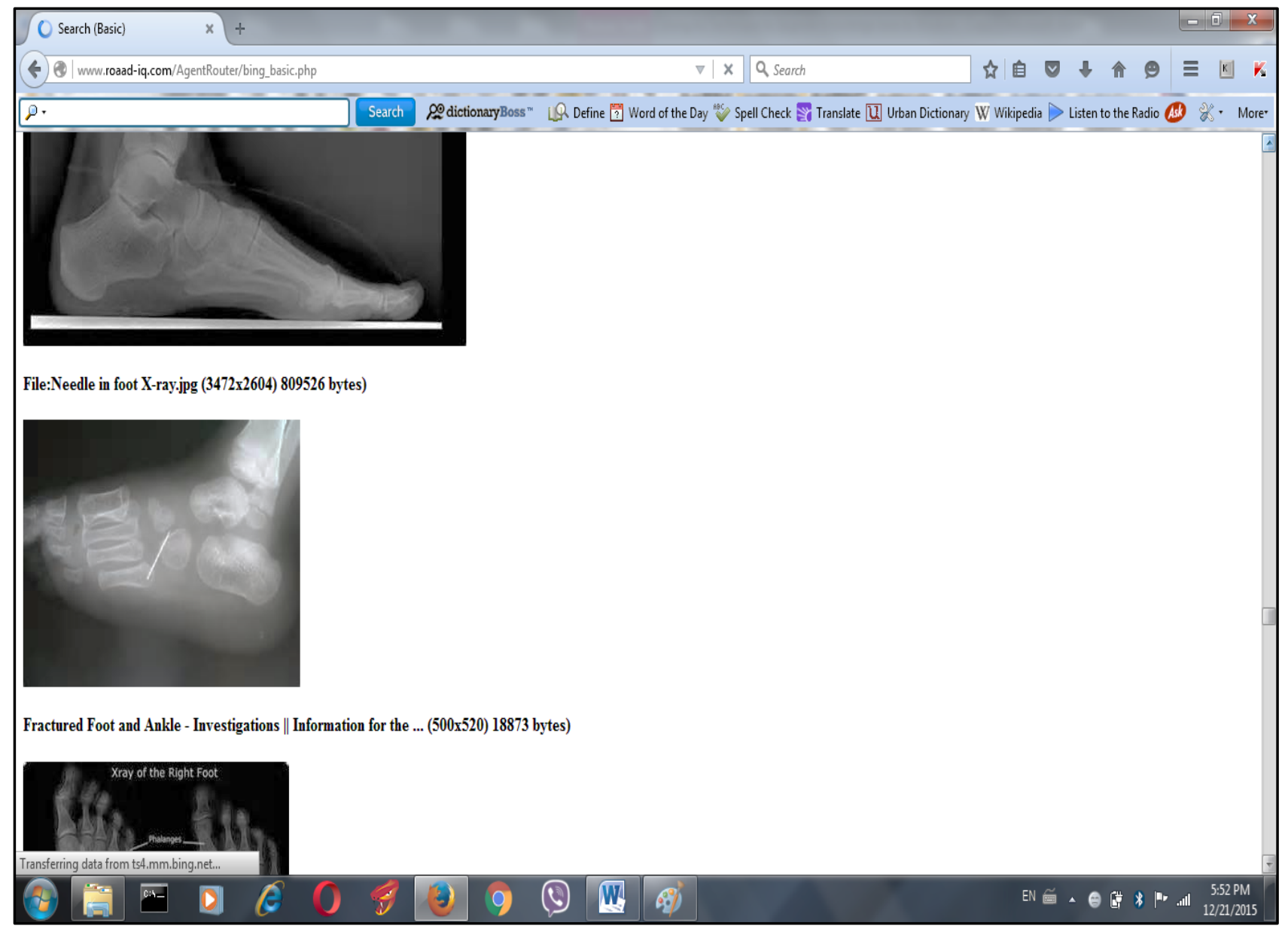

Fig.(4 b), : Continued.

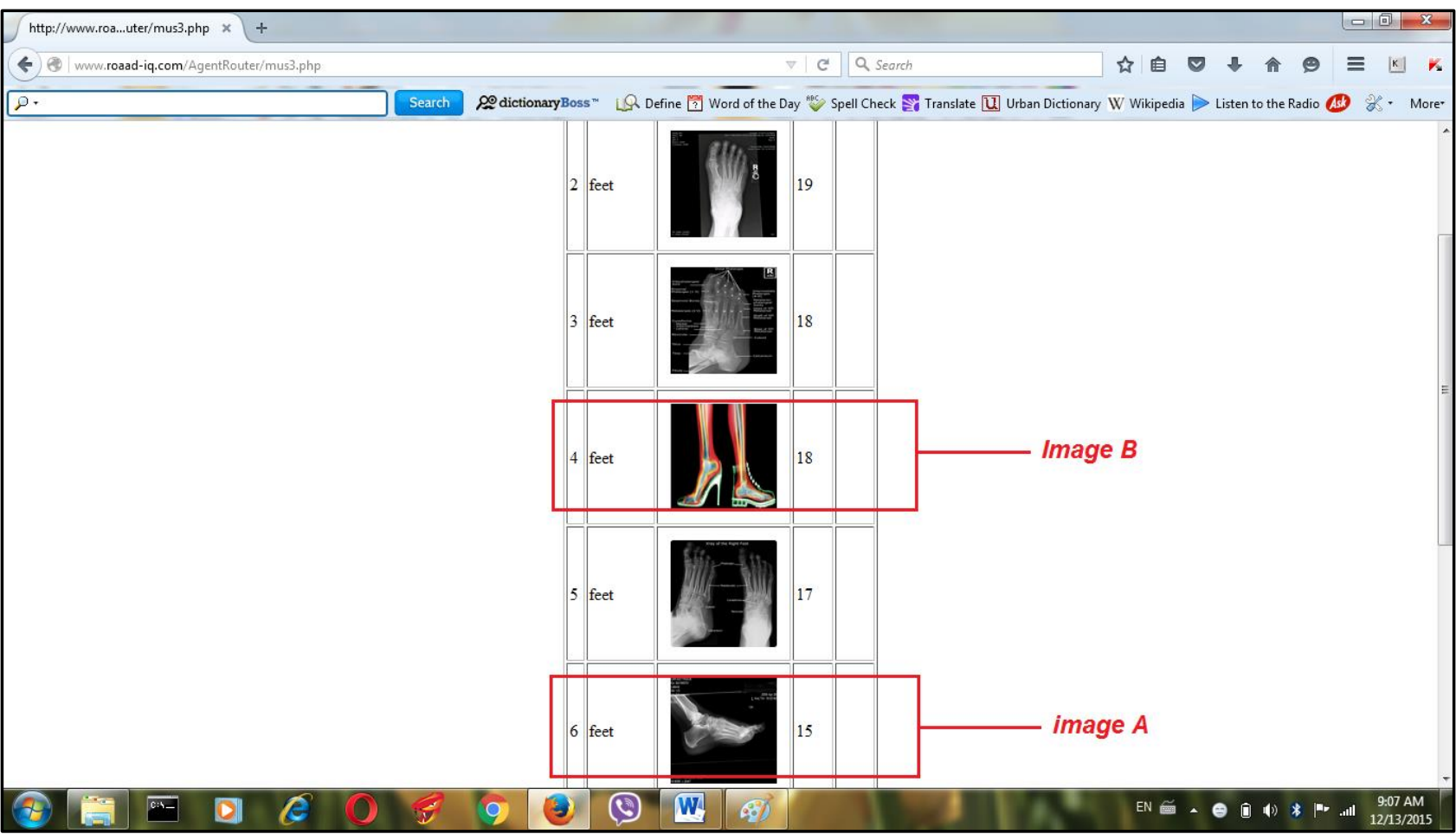

Fig.(5 a) : Images' URLs Results According to submitted Query "feet" in the proposed work (Data Warehouse Searching). 


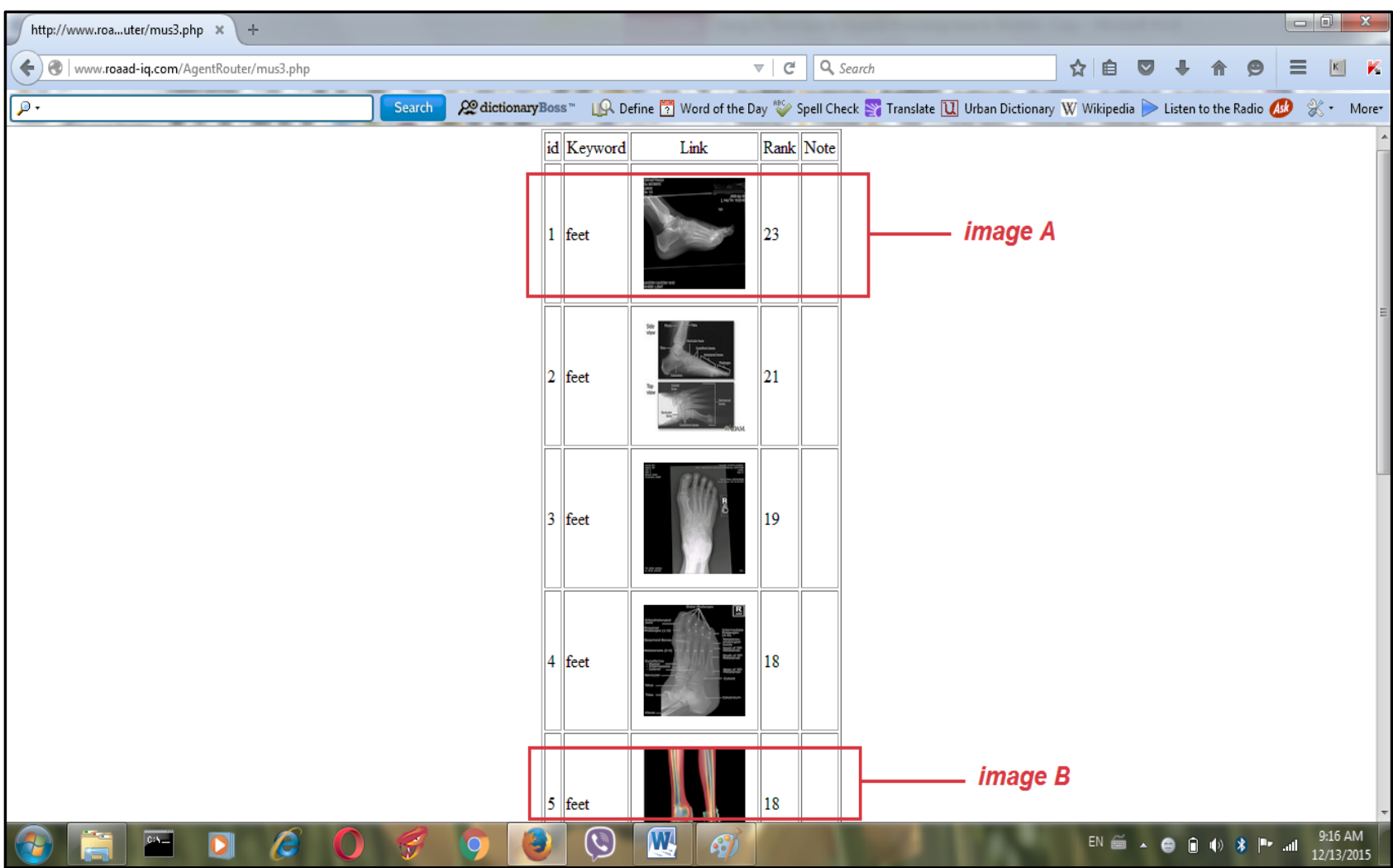

Fig.(5 b) :Continued.

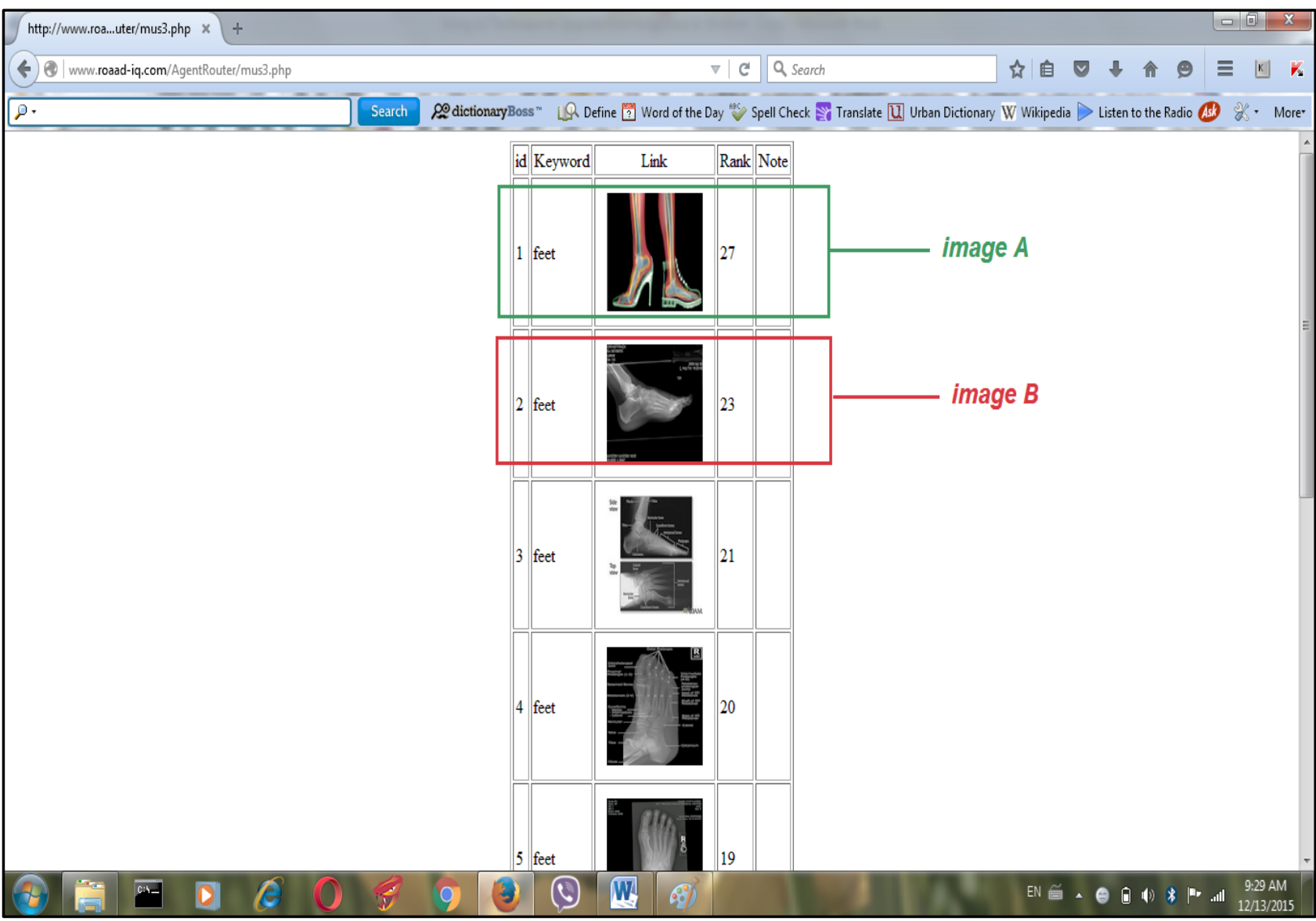

Fig.(5 c) :Continued. 
1. The response by proposed work to this query or anther queries will be customized according to the medical field (radiography), where the query is specialized over the web (Internet) as it illustrates in Figs. (4a, 4b, and 4c).

2. customization in the response is because/reflects of $\mathrm{KBr}$ professional role in presenting knowledge related to user's specialist, where $\mathrm{KBr}$ will work as search engine specialized in the specialist user's field.

3. In addition, $\mathrm{KBr}$ will present knowledge (images) integrated with experience in this field when KB search in the DW. System's results may be changed and different in their ranking related to acquiring and accumulating experiences which leads to change the result depending on the connecting weight with images' URLs with current query by dealing with different and distributed specialists users as it illustrates in Figs. (5a, 5b, and 5c).

4. As a result $\mathrm{KBr}$ will be promoted to presents knowledge upgraded to wisdom level in the medical field to elaborate specialist users' capabilities in making decisions reach wise decisions to be exploited in treatment of diseases cases required deep knowledge and experience in dealing with such cases.

5. $\mathrm{KBr}$ experience is affected by two factors; the long learning period, and number of the specialist users. Increased learning period leads to increase specialist users who use the proposed work (whose $\mathrm{KBr}$ deals with them), and this lead to increase $\mathrm{KBr}$ experience in the medical field, queries, images' URLs in sequence. Thus, the proposed work reliability will increase in presenting knowledge dependent on accumulation of experience (wisdom) which meets users' need.

\section{6- Proposed System Evaluation by Comparing to Related Work}

According to the proposed system policies and aim, $\mathrm{KBr}$ presents web service to the specialist user representing in provide knowledge in the medical field (radiography) upgrade to wisdom level by follow integrated scheme. The scheme representing in aggregate knowledge with experience which acquired and collected from users' experience by dealing $\mathrm{KBr}$ with different and distributed specialist users. $\mathrm{KBr}$ used in the proposed system as a mediator between knowledge resources and specialist user. $\mathrm{KBr}$ provide level of knowledge sustain and elaborate user's capability in making decisions reaching wise decisions to be WKBr. Table (1), presents a comparison between the proposed system and other traditional works in term of measures adopted by this proposed.

Table (1)

Comparison between the Proposed System and Other Traditional works.

\begin{tabular}{|c|c|c|c|}
\hline No. & Attributes & Work's Description & Proposed system \\
\hline 1 & $\begin{array}{l}\text { - Information broker } \\
\text { professional (IB) }\end{array}$ & $\begin{array}{l}\text { - Using IB professional to provide } \\
\text { valuable information to assist } \\
\text { users in decision-making } \\
\text { process. }\end{array}$ & $\begin{array}{l}\text { - Provide knowledge by using } \\
\text { knowledge broker }(\mathrm{KBr}) \text { professional } \\
\text { to assist specialist users in decision- } \\
\text { making process. }\end{array}$ \\
\hline 2 & $\begin{array}{l}\text { - Identification } \\
\text { experience }\end{array}$ & $\begin{array}{l}\text { - Does not support IB professional } \\
\text { with any experience schema to } \\
\text { enrich the provided information } \\
\text { level. }\end{array}$ & $\begin{array}{l}\text { - } \mathrm{KBr} \text { uses an experience scheme to } \\
\text { promote provided knowledge to } \\
\text { wisdom level. }\end{array}$ \\
\hline 3 & - Learning & $\begin{array}{l}\text { - Periodical to identify new classes } \\
\text { of information. }\end{array}$ & - Continues. \\
\hline
\end{tabular}




\section{7- Conclusions}

Based on the implementation of the proposed work and with the presented figures above, several remarks can be concluded:

1. The proposed system is considered a new intelligent system from side of its aim (upgrade knowledge to wisdom level), so a powerful advantage has been gotten in possibility of making decisions reach to wise decisions level.

2. Use of the intelligent agent-based knowledge broker professional as an A.I. technique, has a crucial role in achieving the aim of this work (reaching wisdom), because this professional has many properties similar to human's characteristics (section 2.4) that enables $\mathrm{KBr}$ to develop knowledge in service type that is presented.

3. Use of $\mathrm{KBr}$ in searching process and retrieving the results instead of traditional search process leads to make an efficient search process in a cost effective manner in term of time consuming in the enterprise environment and facilitates it (Figs. (4a,4b)).

4. Use of JADE platform is an important choice in providing the required environment for intelligent agent-based knowledge broker to share experience through socialization scenario.

5. Using DW as a repository by $\mathrm{KBr}$ to store the experience database is an optimal choice, due to warehouse properties of keeping historical data, non-operational, not deleted, and integrated to enable $\mathrm{KBr}$ to achieve the purpose of this research (wisdom), where wisdom is needed to accumulate experience over time (Figs. (5a, $5 b, 5 c))$.

6. The proposed system is flexible and updateable, so it will accept future works to raise its implementation level so as to give an optimal web services.

\section{References}

[1] Anthony Liew, "DIKIW: Data, Information, Knowledge, Intelligence, Wisdom and their Interrelationships", Business Management Dynamics, 2 (10), 49-62, 2013.
[2] Yücel Yilmaz, "Knowledge Management in E-Learning Practices", TOJET: The Turkish Online Journal of Educational Technology, 11 (2), 2012.

[3] Sophie Wrobel, Mohamed Bourimi, Eleni Kosta, Rafael Giménez, and Simon Scerri, "Building Future Generation ServiceOriented Information Broker Networks", (IJACSA) International Journal of Advanced Computer Science and Applications, 4 (11), 2013.

[4] Yossra Hussain, "Agent-Based Program Generation for Database Systems", Ph.D. thesis, Computer Sciences Department of the University of Technology, 2006.

[5] Massimo Paolucci, Julien Soudry, Naveen Srinivasan and Katia Sycara," A Broker for OWL-S Web Services", Extending Web Services Technologies: The Use of MultiAgent Approaches, springer, 2004.

[6] Rancan C., Pesado P. and García-Martínez R., "Toward Integration of Knowledge Based Systems and Knowledge Discovery Systems", JCS\&T, 7 (1), 2007.

[7] Darai D.S., Singh S., Biswas S., "Knowledge Engineering-an Overview", (IJCSIT) International Journal of Computer Science and Information Technologies, 1 (4), 230-234, 2010.

[8] Emberey C.L., Milton N.R., and Vermeulen B., "Application of Knowledge Engineering Methodologies to Support Engineering Design Application Development in Aerospace”, the American Institute of Aeronautics and Astronautics, Inc., AIAA 2007-7708, 2007.

[9] Shiv Kumar Gupta and Ritu Vijay, "Role of Metadata in Data Warehousing for Effective Business Support", Oriental Journal of Computer Science \& Technology, 4 (2), 2011.

[10] Gupta B. M., Kundra R., Gupta S. P., "Information Brokers", Annals of library science and documentation, 30(2), 1983.

[11] Dimitar Christozov, Stefka TolevaStoimenova, "The Role of Information Brokers in Knowledge Management", Online Journal of Applied Knowledge Management, 2 (2), 2014. 
[12] Zahraa Faiz, "Design and Implementation of Multi-Agent System to Perform Electronic Examination System", MSc. Thesis, Computer Sciences department of University of Technology, 2014.

[13] Shaima Sami, "Design and Implementation of Conceptual MultiAgent System to Detect the Threats in the Local Area Network", MSc. Thesis, Computer Sciences Department of University of Technology, 2014.

[14] David Grimshaw, "JADE Administration Tutorial, JADE Architecture Overview", 2010 Available: http://jade.tilab.com/doc/tutorials/JADEAd min/jadeArchitecture.html

[15] Alexander Kolesnikov, "Enterprise Application Development Using Jakarta Tapestry", MSc. Thesis, Glasgow Caledonian University, 2006.

[16] Dr. Abdul Monem S. Rahma and Dr. Jamal F. Tawfeq, "A Knowledge Representation Language for Arabic Semantic Web Using Resources Description Framework", Eng. \& Tech. Journal, 28(18), 2010.

[17] Joep Crompvoets and Bruno Broucker, "Geospatial Information Broker. A new RoleofNational Mapping Agencies", ResearchGate, Gio Information, ISSN: 1857-9000, 1857- 9019, (4), 2015.

[18] James Conklin, Elizabeth Lusk, Megan Harris, and Paul Stolee, "Knowledge Brokers in Knowledge Network: the Case of Seniors Health Research Transfer Network Knowledge Brokers", Implementation Science, BioMed Central, 2013.

[19] Anu Purhonen and Sakari Stenudd, "Runtime Performance Management of Information Broker Based Adaptive Applications", LNCS 6903, 203- 206, 2011.

[20] David Budgen, Mark Turner, Loannis Kotsiopoulos, Fujun Zhu, Michelle Russell, Michael Rigby, Keith Bennett, Pearl Brereton, John Keane, and Paul Layzell, "Managing Healthcare Information: the Role of the Broker", from Grid to Healthgrid T. Solomonides et al. IOS Press, 2005. 